\title{
Microfluidic as Liquid Lens for THz Reconfigurable Antenna and Gain Enhancement With Sensing Application
}

Sepideh Ebrahimi ( $\square$ sepideh.ebrahimi87@yahoo.com )

Azad University

\section{Research Article}

Keywords: antenna, Terahertz, microfluidic, Lens, reconfigurable

Posted Date: July 21st, 2021

DOI: https://doi.org/10.21203/rs.3.rs-727340/v1

License: (a) (i) This work is licensed under a Creative Commons Attribution 4.0 International License.

Read Full License

Version of Record: A version of this preprint was published at Optik on July $1 \mathrm{st}, 2021$. See the published version at https://doi.org/10.1016/j.ijleo.2021.167705. 


\title{
Microfluidic as liquid lens for THz reconfigurable antenna and gain enhancement with sensing application
}

\author{
Sepideh Ebrahimi \\ ${ }^{1}$ Department of Electrical Engineering, Aligudarz Branch, Islamic Azad University, Aligudarz, Iran \\ Corresponding author: sepideh.ebrahimi87@yahoo.com
}

\begin{abstract}
- the lens plays important role in the dielectric resonator antenna (DRA) for increasing the bandwidth and directivity. Also, the liquids like water and ethanol can be noticed for developing the DRA for THz application. In addition, the liquids are interesting because the level and quality of material can be used for designing reconfigurable antenna. For the $\mathbf{T H z}$ spectrum, the liquids can be combined with antenna and microwave devices by Microfluidic technology. In this paper, the suggested antenna is used to enhance the various factors of the antenna and the filling ratio of the channels is noticed to provide reconfigurable antenna. The slot antenna with split ring resonator and meandered feed is used as the basic antenna to provide high Q-factor. Moreover, the results show that this novel structure can be used as sensor for material detecting which is used to recognize various types of liquids by determining the sensitivity of the sensor in the range of 0.5 to $1 \mathrm{THz}$. The proposed sensor is examined for pure water, ethanol and gasoline based on the $2^{\text {nd }}$ order model of Debye.
\end{abstract}

Index Terms - antenna; Terahertz; microfluidic; Lens; reconfigurable

\section{Introduction}

Various methods have been suggested to design reconfigurable antenna for microwave and $\mathrm{THz}$ application [12]. Some methods are electrical, such as PIN diodes [3] and varactor capacitors [4]. Some methods are physical, such as using micro-switches [5] and other types of these antennas are based on materials such as the use of ferrites [6], graphene [7] and liquid crystals [8]. But each of these methods has their own drawbacks and advantages, for example, the use of electrical methods requires separate excitation for PIN diode or varactor $[9,10]$. And in physical methods, an external force needs to activate the switch. For using materials in a reconfigurable antenna, manufacturing problems are often a challenge.

On the other hand, we should notice that in the design of antennas, especially DRA dielectric antennas, the dielectric element plays an effective role in increasing the antenna gain and bandwidth and also miniaturization [11, 12]. Moreover, the effective wavelength of microstrip antennas is proportional to the permittivity of the substrate [13]. Therefore, the use of substances such as water and alcohol, which have a high permittivity, causes great size reduction. So, recently extensive studies have been done on this type of liquid dielectric antennas [14]. One of them was the design of wideband monopole antenna with liquid dielectric in 2006 [15]. In recent years, several models of DRA antennas with a liquid dielectrics and circular polarization have been proposed for wireless applications [16].

In addition, the $\mathrm{THz}$ antenna technology has been developed for various goals such as cancer detection [17] and antenna with different forms have been developed of patch antenna with photonic crystal [18], dipole antenna [19], slot antenna [20], and Yagi antenna [21-22]. However, to detect material under test for the $\mathrm{THz}$ spectrum using metasurface [23] and absorbers [24] are more interesting than antenna. Furthermore, the microfluidic technology has been used for transferring material [25] which can be noticed for sensing and material detection [26-27] and also it can be used for making a lens because of the higher refractive index of liquids such as water.

Moreover, the reconfigurable antennas that can work at multiple frequencies are essential to reduce operating costs in the communications industry. So recently research has been done for using liquid dielectric as a reconfigurable antenna based on a micro pump for controlling the water level [28]. In addition, Using suspended nanoparticles (emulsion) by exciting with an external field, in which a special electric field or magnet can be applied to the liquid dielectric [29,30], and as a result, the polarization of the particles causes a change in the electrical and magnetic conductivity, permittivity and permeability and it can be interesting for future studies [31].

So, based on the previous researches, in this paper a combination of the microfluidic and $\mathrm{THz}$ antenna technology is considered as technique for controlling the antenna behavior including directivity, bandwidth and operation frequency. In this study, the slot antenna is developed for $\mathrm{THz}$ application and then liquid dielectric load based on microfluidic is added to the basic antenna by placing the micro channel over the antenna surface. This antenna shows reconfigurable characteristic by changing the volume of liquids in micro channels. In addition, it can be used for material recognition and in this study, the water, ethanol and gasoline are tested. 


\section{The antenna design and modeling}

The slot antenna is considered as the basic antenna (step 1) which is excited by meandered feed line with the total length and width of 410 and $10 \mu \mathrm{m}$. The result shows this antenna has wide bandwidth and low Q-factor. So, in the second step (step 2) a split ring resonator is added to basic antenna. It reduces the bandwidth of antenna and increases the Q-factor. Finally, the micro channel is placed over the proposed antenna to pass liquid for either enhancing the antenna directivity and bandwidth or detecting material as a sensor in the step 3 .

This antenna is excited by a waveguide port which plays the role of photo-mixer and the simulations have been done with CST microwave studio as a full wave software with open and space boundary for every direction. The lossless quartz with permittivity of 3.75 and the thickness of $10 \mu \mathrm{m}$ is used as the substrate of this antenna and the meandered feed line is placed under the substrate.

A rectangular aperture is made on the ground layer of antenna. Then the SRR is pinpointed in the aperture for concentrating energy in the antenna for reducing bandwidth and Q-factor. Apparently, the gaps in the SRR can make hot spot for trapping energy. The gold is used for metal layer with the height of $0.1 \mathrm{um}$. At last, a thin film of PDMS (polydimethylsiloxane) with permittivity of 2.3 is placed on the split ring with the thickness of $2 \mu \mathrm{m}$ that demands for real experimental fabrication.

The microfluidic with height and width of 18 and $78 \mu \mathrm{m}$ are placed over the PMDS layer. The lossless silicon is selected for the proposed micro channel with permittivity of 11.9. The Fig.1 (a) shows the proposed antenna ground layer with the SRR and the feed line is shown in Fig.1 (b). The 3D view of antenna and microfluidic placement is presented in Fig.1(c) and all dimensions of the proposed antenna are $\mathrm{L}_{1}=$ $200 \mu \mathrm{m}, \mathrm{L}_{2}=100 \mu \mathrm{m}, \mathrm{L}_{3}=55 \mu \mathrm{m}, \mathrm{L}_{4}=40 \mu \mathrm{m}, \mathrm{L}_{5}=100$ $\mu \mathrm{m}, \mathrm{L}_{6}=40 \mu \mathrm{m}, \mathrm{L}_{7}=100 \mu \mathrm{m}, \mathrm{L}_{8}=300 \mu \mathrm{m}, \mathrm{L}_{9}=250 \mu \mathrm{m}$, $\mathrm{L}_{10}=100 \mu \mathrm{m}, \mathrm{L}_{11}=18 \mu \mathrm{m}$ and $\mathrm{L}_{12}=150 \mu \mathrm{m}$.

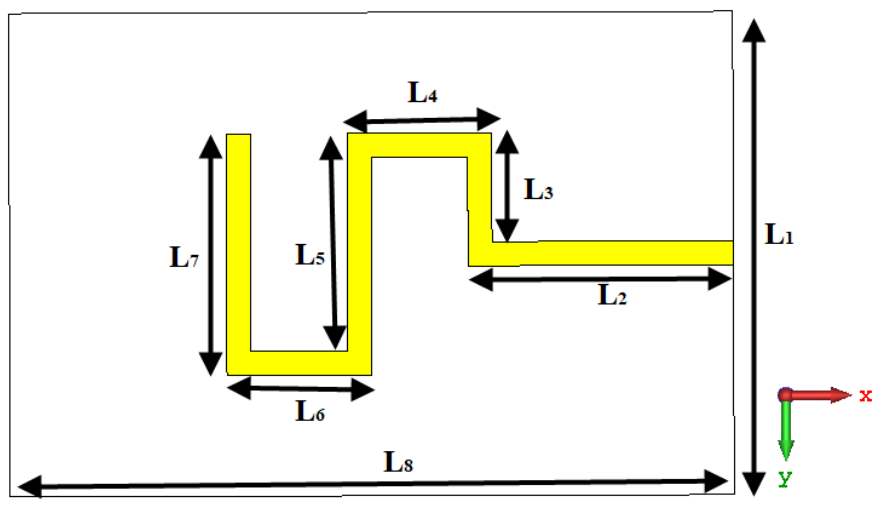

(a)
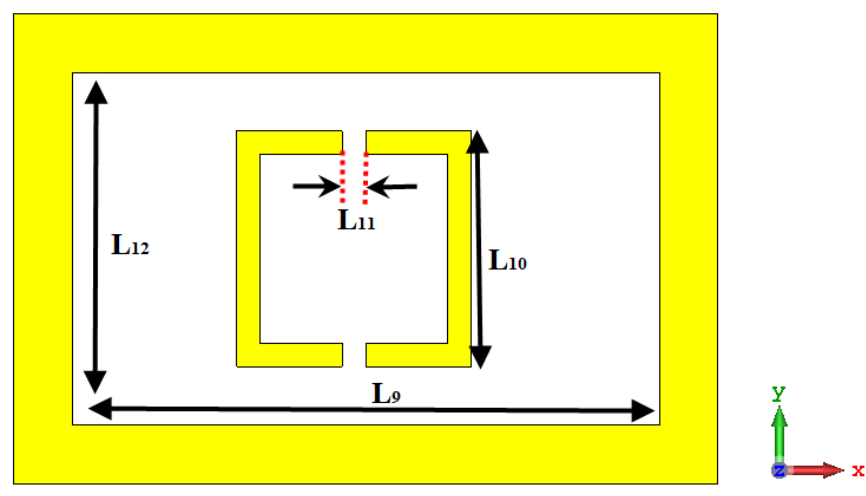

(b)

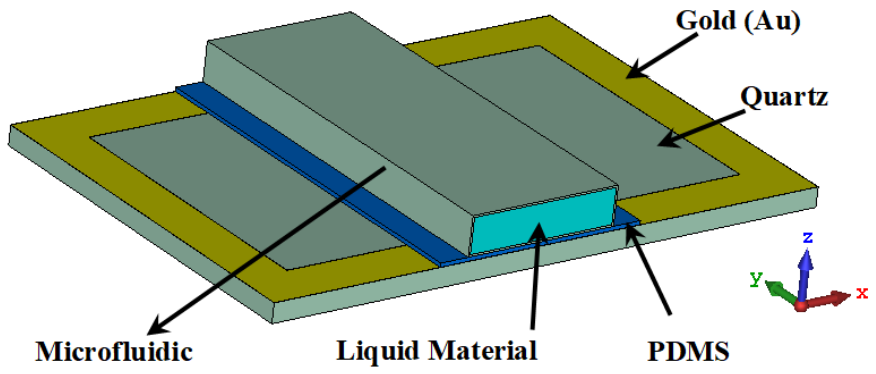

(c)

Fig.1 the geometry for the proposed antenna (a) feed line (b) the ground layer with SRR (c) placement of the Microfluidic over the ground layer

\section{Discussions and Results of the antenna}

The return loss of the antenna in the Step 1 and 2 are checked and presented in Fig.2 in the range of 0.5 to $1 \mathrm{THz}$. As shown here, in the step 1 , the antenna bandwidth in absence of the SRR covers 0.75 to $0.822 \mathrm{THz}$ and it is about $29 \%$. In this case, the minimum value of the return loss is -18 $\mathrm{dB}$. The Q-factor of the antenna can be obtained by Eq.1 where the $f_{0}$ is the operation frequency. The Q-factor for this case is about 16.8 , but when the SRR is added to antenna ground, the antenna bandwidth is reduced to $14.7 \%$. In this case the minimum value return loss is $-24 \mathrm{~dB}$ and antenna Qfactor is 39.1 .

$Q=\frac{f_{0}}{B W_{3 d B}}$

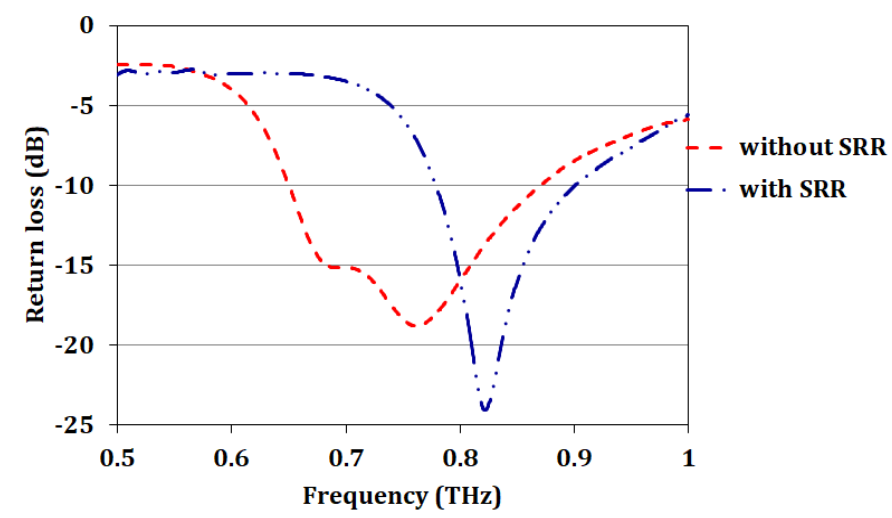

Fig.2 the return loss of the antenna in presence and absence of the SRR

The current distribution for the proposed antenna is presented in Fig.3 at $0.82 \mathrm{THz}$. As shown in Fig.3, the feed 
line coupled with the SRR and the current makes a loop in SRR and also the field concentrates in the SRR which causes the higher Q-factor. On the other hand, as shown in Fig.2, the antenna without SRR has lower frequency because the feed line effective length is longer but when the SRR interacts with the feed line, it reduces the effective length, and therefore a frequency shift to $0.82 \mathrm{THz}$ is visible.

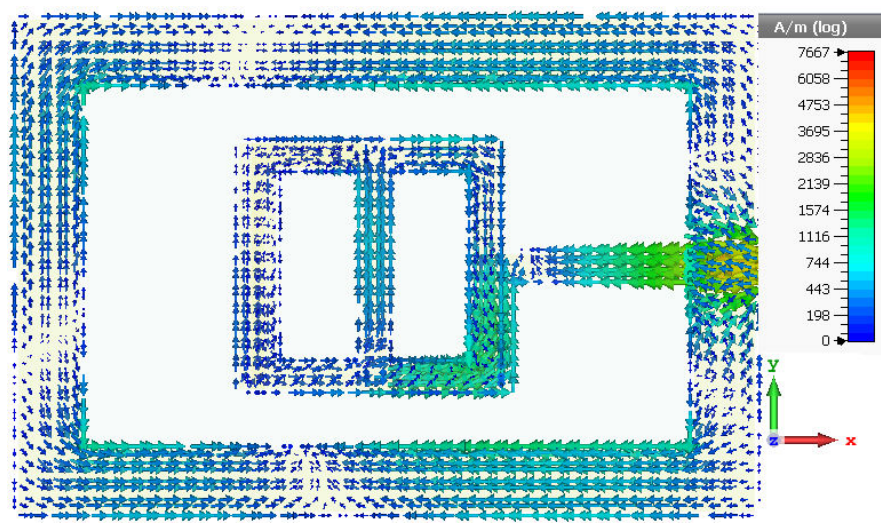

Fig.3 the antenna current distribution at $0.80 \mathrm{THz}$

To realize the effect of the gaps on the antenna Q-factor, the gaps width $\left(\mathrm{L}_{11}\right)$ is checked for 2 to $18 \mu \mathrm{m}$. As shown in Fig.4, the antenna with larger gaps has higher Q-factor, or in other words, the return loss (antenna matching) improved and it reduces to $-31 \mathrm{~dB}$ for $\mathrm{L}_{11}=18 \mu \mathrm{m}$.

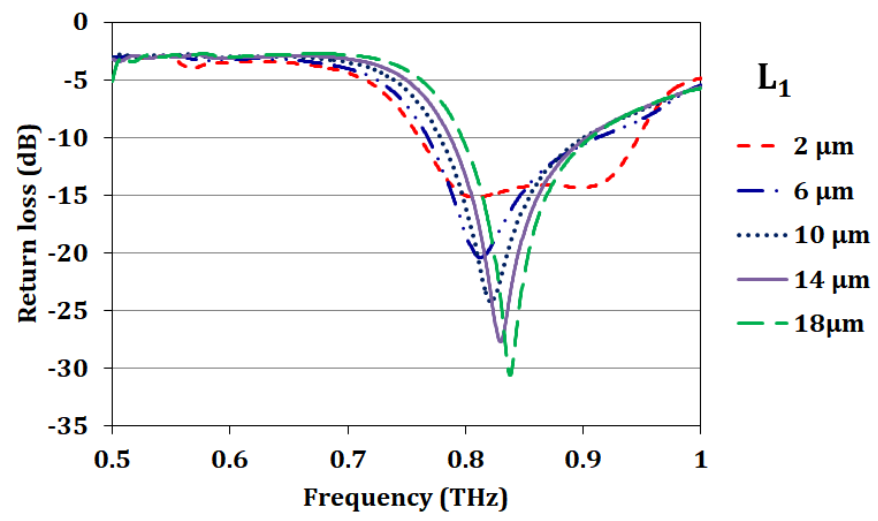

Fig.4 the return loss of the antenna for various SRR's gaps size

Recently various studies have been done for extracting the permittivity of alcohol [32-35], gasoline [34] and water [36-37] in the THz spectrum in the range of 0.5 to $2 \mathrm{THz}$. The Debye second order is used for modeling the liquid material in the $\mathrm{THz}$ spectrum $[34,38-40]$ :

$\varepsilon(\omega)=\varepsilon_{\infty}+\frac{\varepsilon_{S}-\varepsilon_{2}}{1+i \omega \tau_{1}}+\frac{\varepsilon_{2}-\varepsilon_{\infty}}{1+i \omega \tau_{2}}$

The $\varepsilon_{\infty}, \varepsilon_{S}, \varepsilon_{2}, \tau_{1}$ and $\tau_{2}$ are temperature dependent of high frequency permittivity, static dielectric constant, intermediate frequency limit, slow relaxation time, and fast relaxation time.
Table.1 $2^{\text {nd }}$ order Debye model coefficients for several types of alcohol and water

\begin{tabular}{cccccc}
\hline & $\varepsilon_{\infty}$ & $\varepsilon_{s}$ & $\varepsilon_{2}$ & $\tau_{1}[\mathrm{Ps}]$ & $\tau_{2}[\mathrm{Ps}]$ \\
\hline water & 3.23 & 78.3 & 5.29 & 7.92 & 0.182 \\
ethanol & 2.12 & 24.35 & 4.44 & 163 & 8.97 \\
Gasoline & 1.96 & 2.36 & 2.06 & 3.18 & 0.05 \\
\hline
\end{tabular}

The real and imaginary part of the permittivity of the pure water, ethanol and gasoline are presented in Fig.5 which is used in the microfluidic channel for sensing.

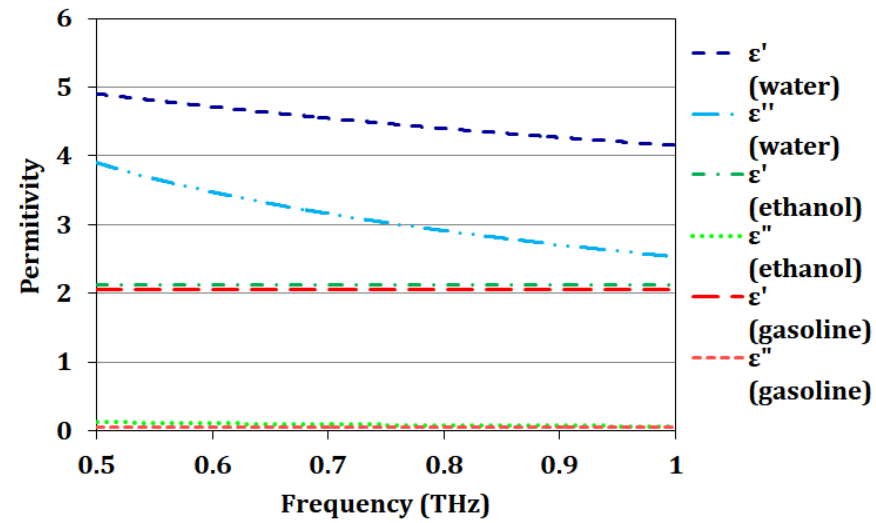

Fig.5 the real and imaginary permittivity of the water, ethanol and gasoline

The return loss of the antenna for the pure water, ethanol and gasoline are compared with the vacuum channel for $\mathrm{L}_{11}=$ $18 \mu \mathrm{m}$. By filling the channel with the liquid, the operation frequency shifts to lower frequency and in addition, the loss which is made by the imaginary part of the liquid impact on the level of the return loss where the return loss for the proposed empty sensor channel is $-33 \mathrm{~dB}$ and this value is changed to $-31 \mathrm{~dB}$ for the gasoline and ethanol and the water is known as a lossy material thus the return loss value is obtained $-22 \mathrm{~dB}$ at $0.8 \mathrm{THz}$

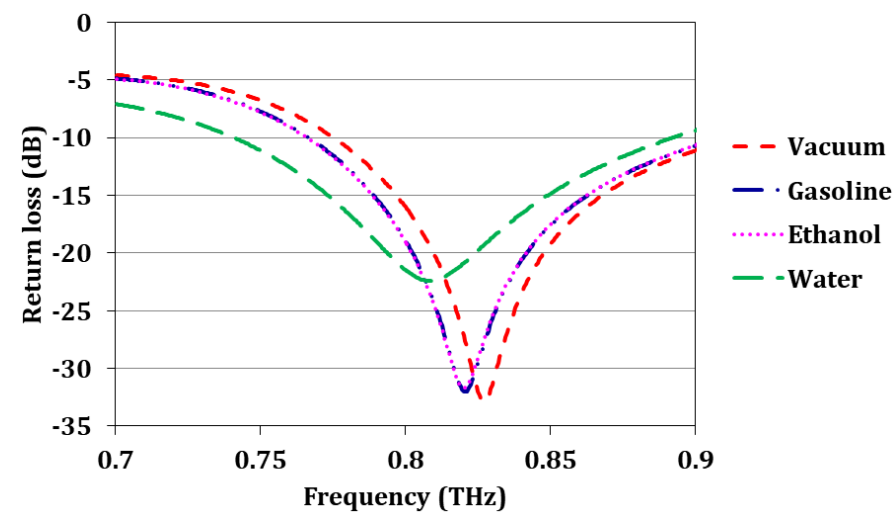

Fig.6 the return loss of the antenna for various material for sensing

The comparisons between three materials are presented in the Table.2. The Q-factor, BW, FOM and sensitivity are considered for recognizing the material in the microfluidic 
channel. For calculating the sensitivity and FOM the Eq.3 and 4 can be used where the $\Delta f$ is the frequency shift, $\Delta \mathrm{n}$ is the refractive index variation and the FWHM is Full width at half maximum of the return loss.

$$
\begin{aligned}
& S=\frac{\Delta f}{\Delta n} \\
& F O M=\frac{S}{F W H M}
\end{aligned}
$$

Table.2 comparing the sensor with different materials

\begin{tabular}{lcccc}
\hline & Q-factor & BW & $\begin{array}{c}\text { FOM } \\
\text { RIU }^{-1}\end{array}$ & $\begin{array}{c}\text { Sensitivity } \\
(\text { GHz/RIU) }\end{array}$ \\
\hline Gasoline & $\mathbf{8 6 . 3 6}$ & $17.15 \%$ & 1554 & 14.77 \\
Ethanol & $\mathbf{8 6 . 8 4}$ & $17.31 \%$ & 1534 & 14.58 \\
Water & 23.1 & $18.49 \%$ & 441 & 15.44 \\
\hline
\end{tabular}

As shown in Table.2, the material under test can be recognizing by their effect on antenna parameters. Here, the water makes a great frequency shift, so despite of higher $\Delta \mathrm{n}$, it has sensitivity of $15.44 \mathrm{GHz} / \mathrm{RIU}$ ), but because of wide bandwidth and lower Q-factor, the FOM shows reduction in comparison the ethanol and gasoline.

As a result, Fig.6 shows that antenna with filled micro channel by water has wider bandwidth in comparison with gasoline and ethanol. Thus this material can be used to develop this antenna by adding a few new channels to the surface of the antenna. The implementing three channels over the proposed antenna filled with water or vacuum is presented in Fig.7. So, here there cases are selected for examining the effect of the water on antenna directivity, bandwidth and frequency shift. In the first case all channel are filled by water. In the second case, the Ch.1 is assumed vacuum and only Ch.2 and Ch. 3 are filled by water. Finally in the third case, only the Ch.2 is filled by water.

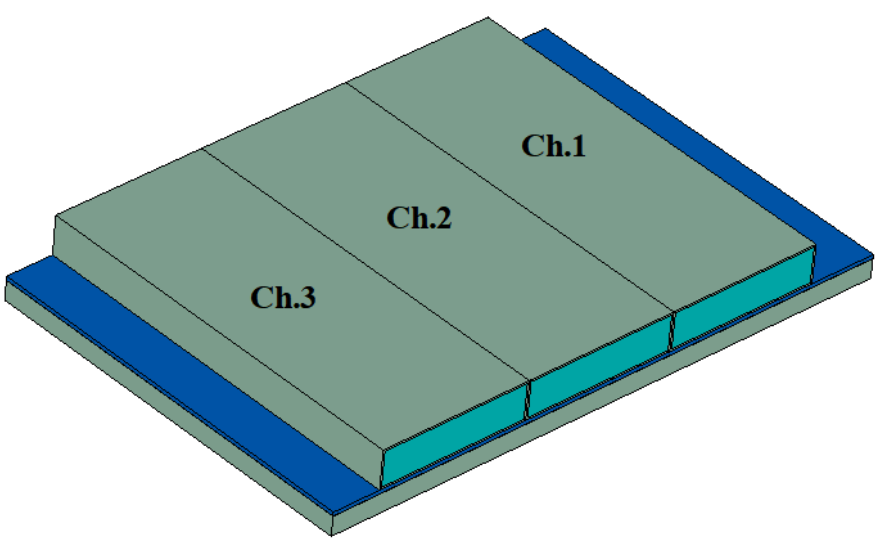

Fig.7 the implementing three channels over the proposed antenna filled with water or vacuum
The return loss of the antenna for three cases is presented in Fig.8. As shown in Fig.8, the antenna operation frequency shifts to from 0.8 to $0.73 \mathrm{THz}$ by increasing the volume of the water in the channel, but water loss reduces the Q-factor of the antenna. On the other hand, the bandwidth of antenna increases from $18.65 \%$ to $49 \%$ from the case 1 to case 3 .So for $\mathrm{THz}$ wireless application, for increasing the band width of the antenna, the liquid dielectric can be used as a solution. Moreover, as this antenna has reconfigurable characteristic to allocate special frequency for sending and receiving difference volume of the liquid can be used.

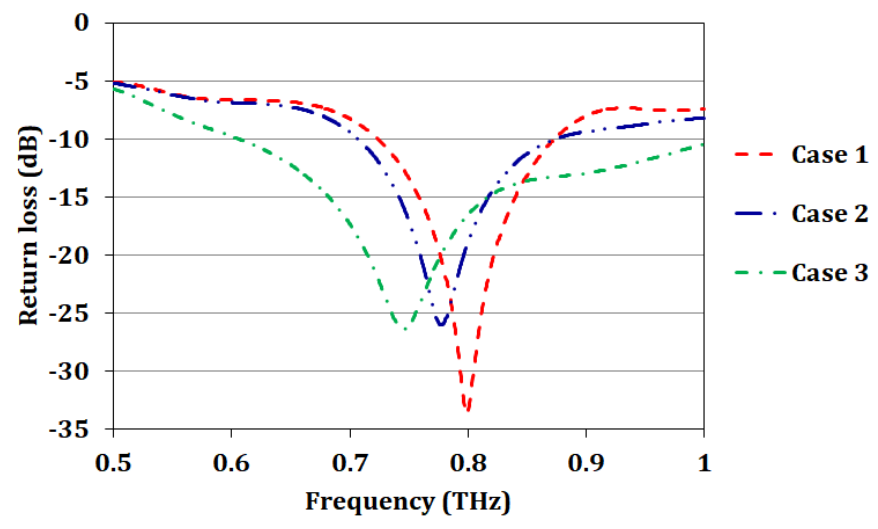

Fig.8 return loss of the antenna for three cases with various volume of water in channels

The directivity of the antenna in three cases are presented in Fig. 9 and for the case 1 , the antenna directivity is $4.69 \mathrm{dBi}$ and the antenna directivity increases to $5.22 \mathrm{dBi}$ for the case 2 when the Ch.2 and Ch.3 are filled by water. When all channels are field by the water, the directivity increases to $5.43 \mathrm{dBi}$.

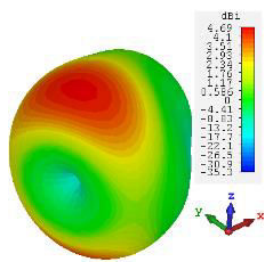

(a)

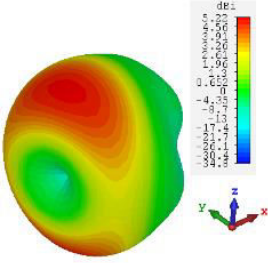

(b)

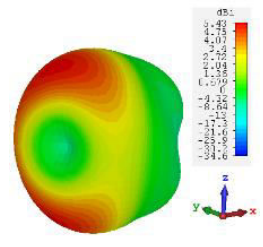

(c)
Fig.9 the directivity of the antenna for three cases (a) case 3: Ch.2 is filled (b) case 2 : Ch. 2 and Ch.3 are filled (c) case 1: all channel filled

\section{Conclusion}

In this paper, combination of the microfluidic structure with slot antenna was considered for sensing and recognizing the pure water, ethanol and gasoline based on the frequency shift and Q-factor. In addition various topologies of the micro channel were suggested to use the microfluidic technique with water for increasing the antenna bandwidth and gain besides designing a reconfigurable antenna in the $\mathrm{THz}$ spectrum. Therefore, combining the microfluidic technique and antenna can be developed for sensing and communication application at the same time. 


\section{References:}

[1] Heydari, Samaneh, Kioumars Pedram, Zeeshan Ahmed, and Ferdows B. Zarrabi. "Dual band monopole antenna based on metamaterial structure with narrowband and UWB resonances with reconfigurable quality." AEU-International Journal of Electronics and Communications 81 (2017): 92-98.

[2] Kazemi, Fatemeh. "Dual band compact fractal $\mathrm{THz}$ antenna based on CRLH-TL and graphene loads." Optik 206 (2020): 164369.

[3] Tripathi, Shivesh, Nagendra P. Pathak, and Manoranjan Parida. "A dual feed PIN diode based switchable multiband planar meandered antenna for intelligent transportation system application." International Journal of RF and Microwave Computer-Aided Engineering 29, no. 11 (2019): e21936.

[4] Row, Jeen-Sheen, and Jia-Fu Tsai. "Frequency-reconfigurable microstrip patch antennas with circular polarization." IEEE Antennas and Wireless Propagation Letters 13 (2014): 1112-1115.

[5] Haupt, Randy L., and Michael Lanagan. "Reconfigurable antennas." IEEE Antennas and Propagation Magazine 55, no. 1 (2013): 49-61.

[6] Lou, Qun, Rui-xin Wu, Fan-guang Meng, and Yin Poo. "Realizing frequency reconfigurable antenna by ferrite-loaded half-mode SIW." Microwave and Optical Technology Letters 59, no. 6 (2017): 1365-1371.

[7] Dragoman, Mircea, Dan Neculoiu, Alina-Cristina Bunea, George Deligeorgis, Martino Aldrigo, D. Vasilache, Adrian Dinescu et al. "A tunable microwave slot antenna based on graphene." Applied Physics Letters 106, no. 15 (2015): 153101.

[8] Anil Kumar, Tirunagari, Yalavarthi Usha Devi, Boddapati TP Madhav, Sarat K. Kotamraju, and Vullanki Rajesh. "High gain flexible liquid crystal polymer based 8-element printed antenna for millimetric wave applications." International Journal of RF and Microwave ComputerAided Engineering 29, no. 7 (2019): e21744.

[9] Seo, Dongcheol, and Youngje Sung. "Reconfigurable square ring antenna for switchable circular polarisation." Electronics Letters 51, no. 6 (2015): 438-440.

[10] Kim, Ilkyu, Byung-chul Park, and Jeong-Hae Lee. "Varactor diode integrated dipole-EBG base-station antenna: Enhancing tilted radiation pattern." Microwave and Optical Technology Letters 57, no. 8 (2015): 1794-1799.

[11] Hu, Peng Fei, Yong Mei Pan, Kwok Wa Leung, and Xiu Yin Zhang. "Wide-/dual-band omnidirectional filtering dielectric resonator antennas." IEEE Transactions on Antennas and Propagation 66, no. 5 (2018): 2622-2627.

[12] Pedram, Kumars, Mohammad Naderi, Fereshteh Sadat Jafari, and Ferdows B. Zarrabi. "Compact quad-band second harmonic antenna based on metamaterial DRA load." Microwave and Optical Technology Letters 61, no. 8 (2019): 1938-1944.

[13] Wong, Kin-Lu. Compact and broadband microstrip antennas. Vol. 168 John Wiley \& Sons, 2004

[14] Song, Chaoyun, Elliot L. Bennett, Jianliang Xiao, Tianyuan Jia, Rui Pei, Kwai-Man Luk, and Yi Huang. 'Passive beam-steering gravitational liquid antennas." IEEE Transactions on Antennas and Propagation 68, no. 4 (2019): 3207-3212.

[15] Fayad, H., and P. Record. "Broadband liquid antenna." Electronics Letters 42, no. 3 (2006): 133-134.

[16] [Ren, Jian, Zhao Zhou, Zhao Hui Wei, Hao Ming Ren, Zhe Chen, Ying Liu, and Ying Zeng Yin. "Radiation Pattern and Polarization Reconfigurable Antenna Using Dielectric Liquid." IEEE Transactions on Antennas and Propagation (2020).

[17] Poorgholam-Khanjari, Shima, and Ferdows B. Zarrabi. "Reconfigurable Vivaldi $\mathrm{THz}$ antenna based on graphene load as hyperbolic metamaterial for skin cancer spectroscopy." Optics Communications 480 (2021): 126482.

[18] Nejati, Ameneh, Ramezan Ali Sadeghzadeh, and Fatemeh Geran. "Effect of photonic crystal and frequency selective surface implementation on gain enhancement in the microstrip patch antenna at terahertz frequency." Physica B: Condensed Matter 449 (2014): 113 120.

[19] Sadeghzadeh, Ramezan Ali, and Ferdows B. Zarrabi. "Metamaterial Fabry-Perot cavity implementation for gain and bandwidth enhancement of THz dipole antenna." Optik 127, no. 13 (2016): 5181-5185.

[20] Kazemi, Fatemeh. "High Q-factor compact and reconfigurable $\mathrm{THz}$ aperture antenna based on graphene loads for detecting breast cancer cells." Superlattices and Microstructures 153 (2021): 106865.

[21] Poorgholam-Khanjari, Shima, Ferdows B. Zarrabi, and Saughar Jarchi. "Compact and wide-band Quasi Yagi-Uda antenna based on periodic grating ground and coupling method in terahertz regime." Optik 203 (2020): 163990.

[22] Zarrabi, Ferdows B., Mir Mohsen Seyedsharbaty, Zeeshan Ahmed, Afsaneh Saee Arezoomand, and Samaneh Heydari. "Wide band yagi antenna for terahertz application with graphene control." Optik 140 (2017): 866-872.

[23] Cheng, Ruijian, Ling Xu, Xin Yu, Liner Zou, Yun Shen, and Xiaohua Deng. "High-sensitivity biosensor for identification of protein based on terahertz Fano resonance metasurfaces." Optics Communications 473 (2020): 125850.

[24] Poorgholam-Khanjari, Shima, Zahra Razavi, and Ferdows B. Zarrabi. "Reconfigurable optical rectangular particle array absorber based on metal-DNA-metal structure as a refractive index sensor and optical switch." Optics Communications 489 (2021): 126866.

[25] Lue, Niyom, Gabriel Popescu, Takahiro Ikeda, Ramachandra R. Dasari, Kamran Badizadegan, and Michael S. Feld. "Live cell refractometry using microfluidic devices." Optics letters 31, no. 18 (2006): 2759-2761.

[26] Faustino, Vera, Susana O. Catarino, Rui Lima, and Graça Minas. "Biomedical microfluidic devices by using low-cost fabrication techniques: A review." Journal of biomechanics 49, no. 11 (2016): 2280-2292.

[27] Li, Xiangjun, Jian Song, and John XJ Zhang. "Design of terahertz metaldielectric-metal waveguide with microfluidic sensing stub." Optics Communications 361 (2016): 130-137.

[28] Xing, Lei, Qian Xu, Jiajia Zhu, Yongjiu Zhao, Chaoyun Song, and Yi Huang. "A high-efficiency wideband frequency-reconfigurable water antenna with a liquid control system: usage for VHF and UHF applications." IEEE Antennas and Propagation Magazine (2019): 0-0.

[29] Fayad, H., and P. Record. "Broadband liquid antenna." Electronics Letters 42, no. 3 (2006): 133-134.

[30] Motovilova, Elizaveta, and Shao Ying Huang. "A review on reconfigurable liquid dielectric antennas." Materials 13, no. 8 (2020): 1863.

[31] Ren, Jian, Zhao Zhou, Zhao Hui Wei, Hao Ming Ren, Zhe Chen, Ying Liu, and Ying Zeng Yin. "Radiation pattern and polarization reconfigurable antenna using dielectric liquid." IEEE Transactions on Antennas and Propagation 68, no. 12 (2020): 8174-8179.

[32] Yomogida, Yoshiki, Yuki Sato, Ryusuke Nozaki, Tomobumi Mishina, and Jun'ichiro Nakahara. "Dielectric study of normal alcohols with $\mathrm{THz}$ time-domain spectroscopy." Journal of Molecular Liquids 154, no. 1 (2010): 31-35.

[33] Saha, Shimul C., James P. Grant, Yong Ma, A. Khalid, Feng Hong, and David RS Cumming. "Terahertz frequency domain spectroscopy for polar alcohol." In 2011 International Conference on Infrared, Millimeter, and Terahertz Waves, pp. 1-2. IEEE, 2011.

[34] Arik, Enis, Hakan Altan, and Okan Esenturk. "Dielectric properties of ethanol and gasoline mixtures by terahertz spectroscopy and an effective method for determination of ethanol content of gasoline." The Journal of Physical Chemistry A 118, no. 17 (2014): 3081-3089.

[35] Jepsen, Peter Uhd, Jens Kristian Jensen, and Uffe Møller. "Characterization of aqueous alcohol solutions in bottles with $\mathrm{THz}$ reflection spectroscopy." Optics express 16, no. 13 (2008): 9318-9331.

[36] Liebe, Hans J., George A. Hufford, and Takeshi Manabe. "A model for the complex permittivity of water at frequencies below 1 THz." International Journal of Infrared and Millimeter Waves 12, no. 7 (1991): 659-675.

[37] Ellison, W. J. 'Permittivity of pure water, at standard atmospheric pressure, over the frequency range $0-25 \mathrm{THz}$ and the temperature range 0-100 C." Journal of physical and chemical reference data 36, no. 1 (2007): 1-18.

[38] Koeberg, Mattijs, Chien-Ching Wu, Doseok Kim, and Mischa Bonn. "THz dielectric relaxation of ionic liquid: water mixtures." Chemical physics letters 439, no. 1-3 (2007): 60-64.

[39] Poorgholam-Khanjari, Shima, Ahmad Hatami, and Ferdows B. Zarrabi. "Compact antenna based on split ring resonator as high Q-factor antenna for liquid permittivity measurements." Frequenz (2021).

[40] Møller, Uffe, David G. Cooke, Koichiro Tanaka, and Peter Uhd Jepsen. "Terahertz reflection spectroscopy of Debye relaxation in polar liquids." JOSA B 26, no. 9 (2009): A113-A125. 